\title{
Nanotopographical surfaces for regulating cellular mechanical behaviors investigated by atomic force microscopy
}

\author{
Mi Li ${ }^{1,2 *}$, Ning $\mathrm{Xi}^{3}$, Yuechao Wang ${ }^{1,2}$, Lianqing $\mathrm{Liu}^{1,2^{*}}$ \\ ${ }^{1}$ State Key Laboratory of Robotics, Shenyang Institute of Automation, Chinese Academy of Sciences, Shenyang 110016 , China \\ ${ }^{2}$ Institutes for Robotics and Intelligent Manufacturing, Chinese Academy of Sciences, Shenyang 110016, China \\ ${ }^{3}$ Department of Industrial and Manufacturing Systems Engineering, The University of Hong Kong, Hong Kong, China \\ *Corresponding authors
}

Tel: +86-24-23970540; Fax: +86-24-23970021; Email: limi@sia.cn; lqliu@sia.cn

Number of Pages: 8

Number of Figures: 11 


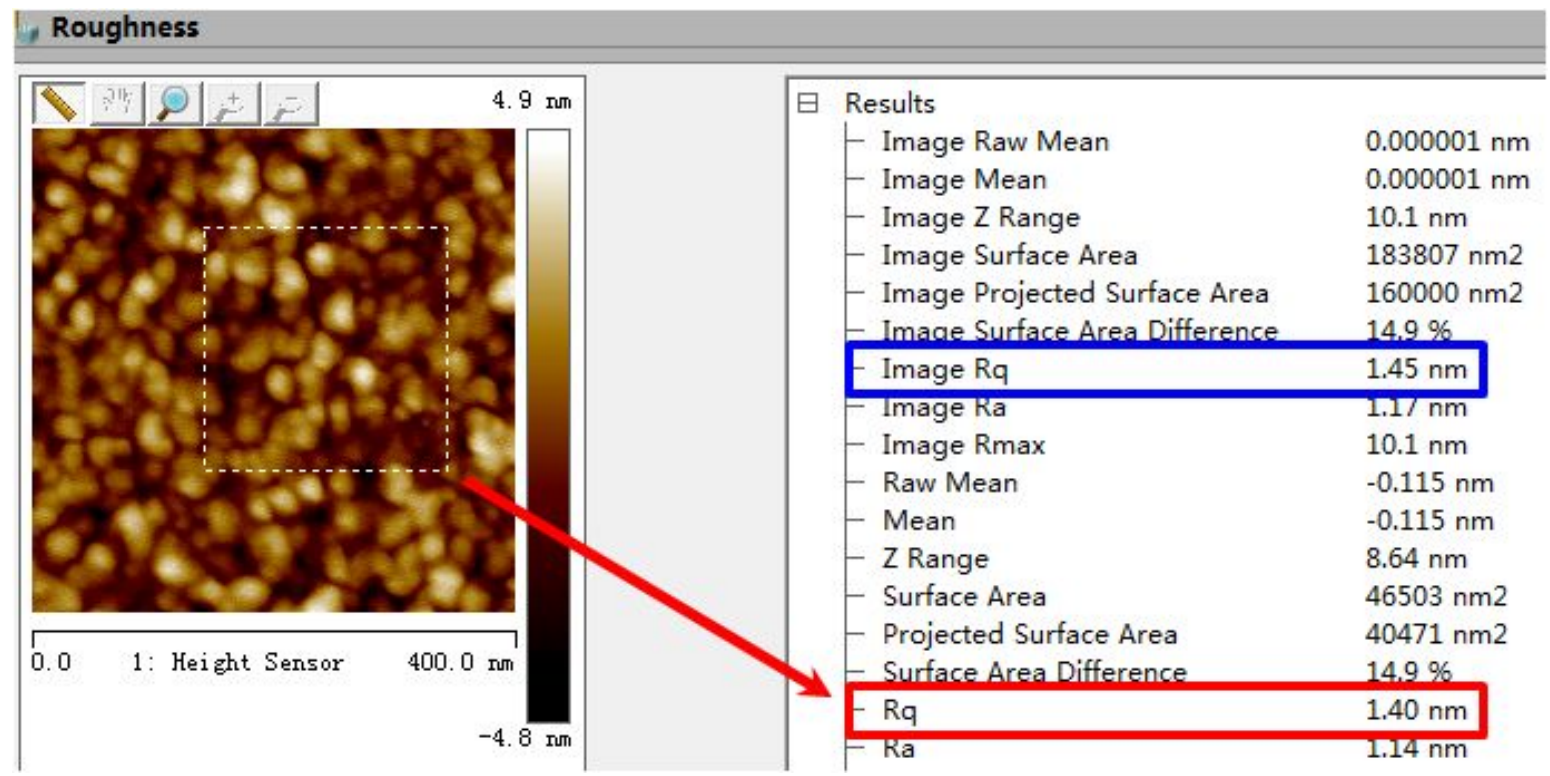

Figure S1 Extracting surface roughness of decellularized substrates by the offline AFM software Nanoscope Analysis (Bruker, Santa Barbara, CA, USA). The "Image $\mathrm{R}_{\mathrm{q}}$ " indicates the root-mean-square roughness $R_{q}$ of the whole image, while the " $\mathrm{R}_{\mathrm{q}}$ " indicates the root-mean-square roughness $R_{q}$ of the selected region (denoted by the white dashed square) of the image.
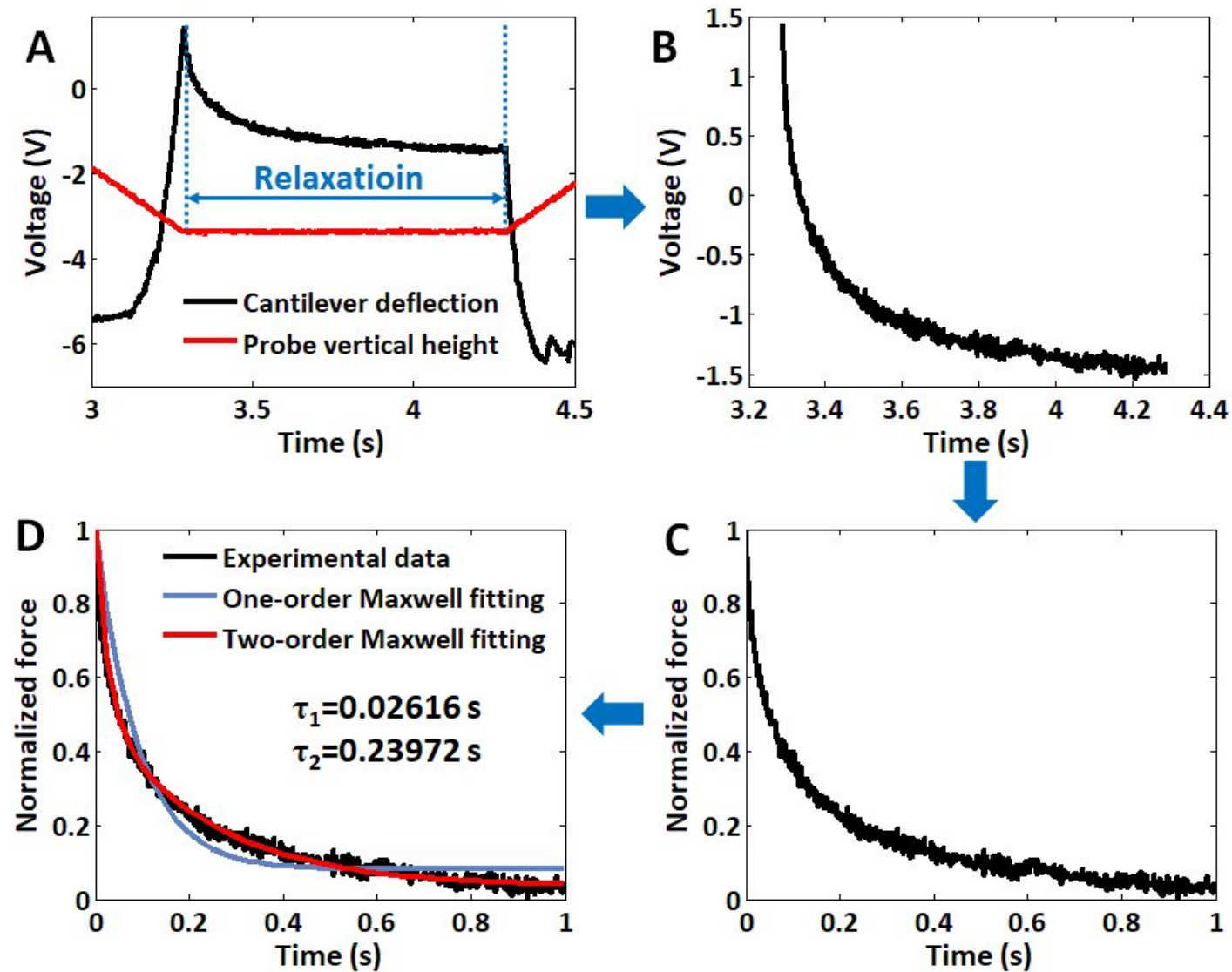

Figure S2 Process of measuring cellular relaxation times. (A) Original relaxation curve (black curve) obtained during the vertical approachdwell-retract process of AFM tip. The red curve corresponds to the vertical displacement of AFM probe. During the stage of dwelling (the vertical position of AFM probe kept unchanged), cellular relaxation was observed, denoted by the blue arrow. (B) The relaxation curve extracted from the experimental curve. (C) The normalized relaxation curve. (D) Fitting the normalized relaxation curve with two-order Maxwell model yields two relaxation times $\left(\tau_{1}\right.$ and $\left.\tau_{2}\right)$. While one-order Maxwell model does not fit the relaxation curve well. 

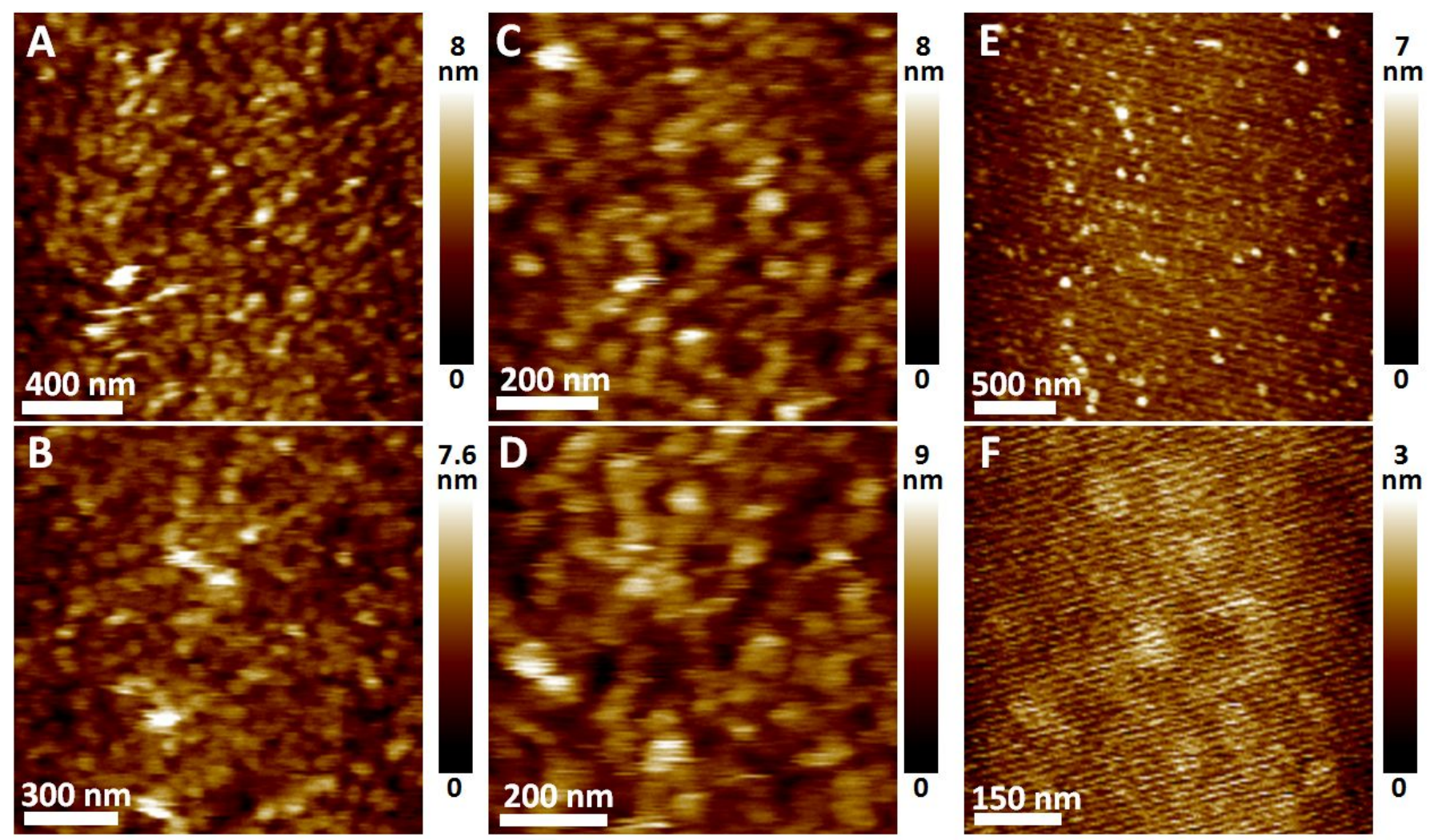

Figure S3 AFM imaging of decellularized glass slides (growing C2C12 cells) in pure water. (A, B) Large scan size imaging. (C, D) Small scan size imaging. (E, F) AFM images of fresh glass slides (without growing cells) in water for control.

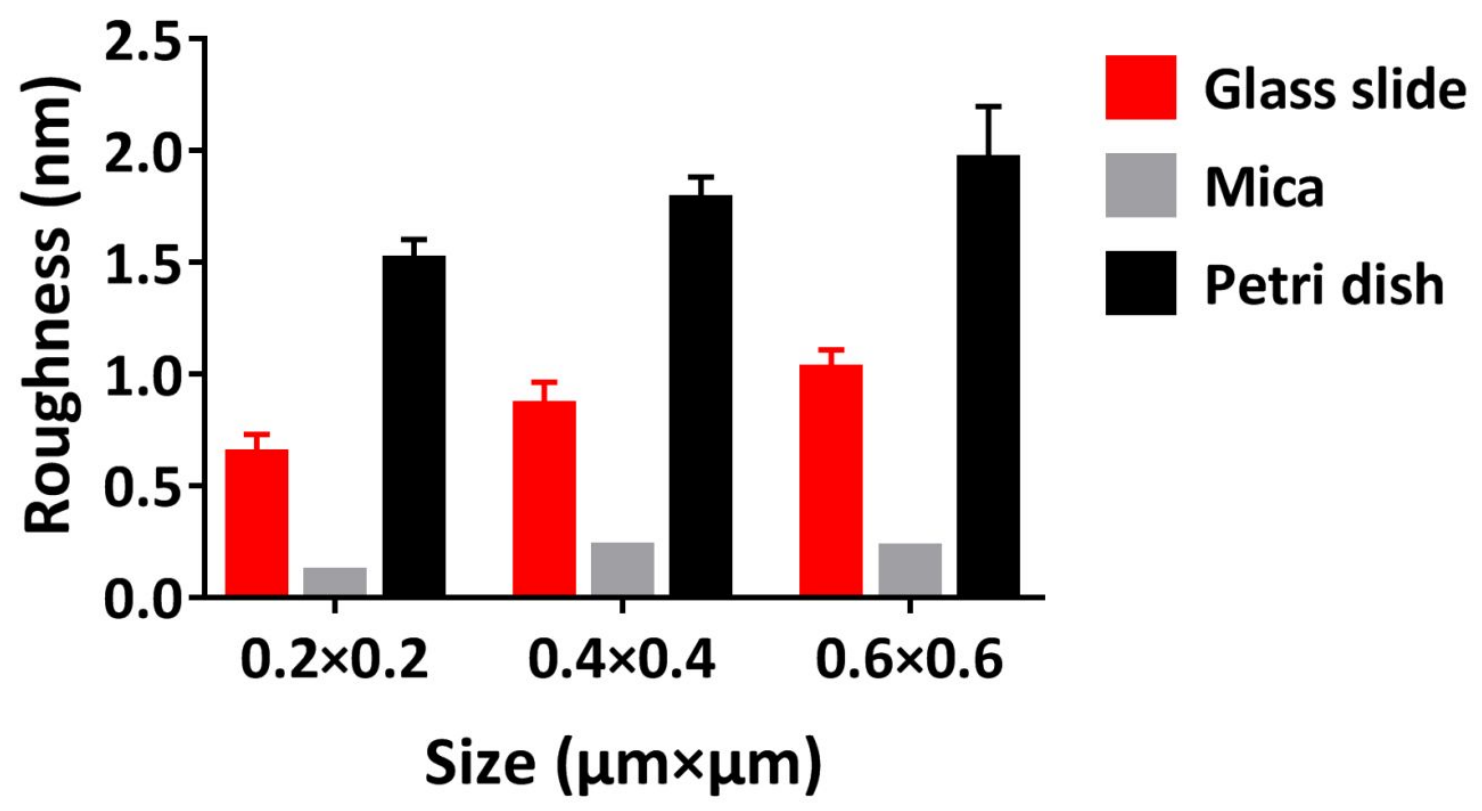

Figure S4 Roughness of bare substrates without growing cells. 

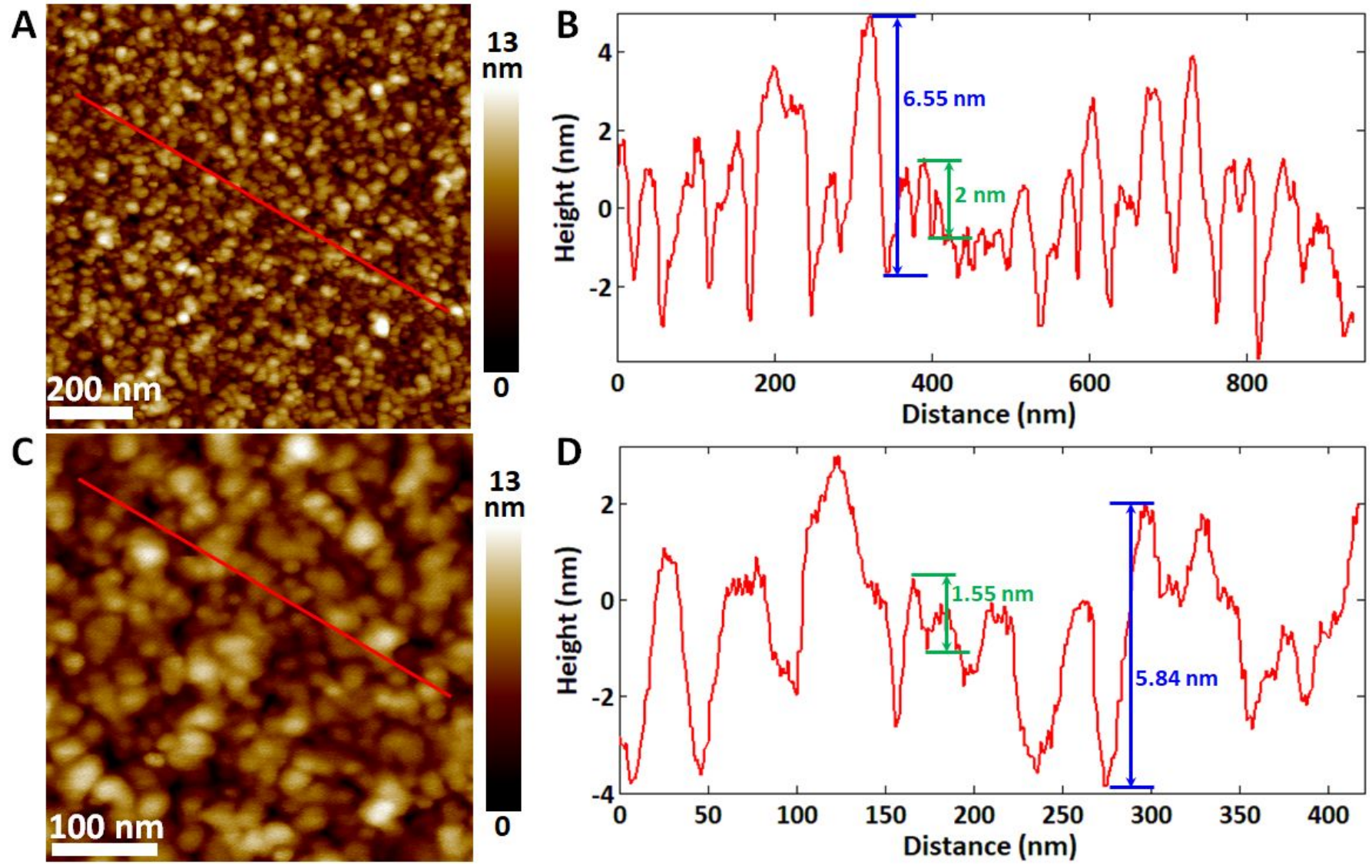

Figure S5 Analyzing the sizes of nanogranular structures on the decellularized substrates. (A) AFM height image of the decellularized substrate obtained by large-size scan imaging. (B) Section profile curve taken along the red line in (A). (C) AFM height image of the decellularized substrate obtained by small-size scan imaging. (D) Section profile curve taken along the red line in (C). The green arrows typically denote the size of small nanogranular feature, while the blue arrows typically denote the size of large nanogranular feature.
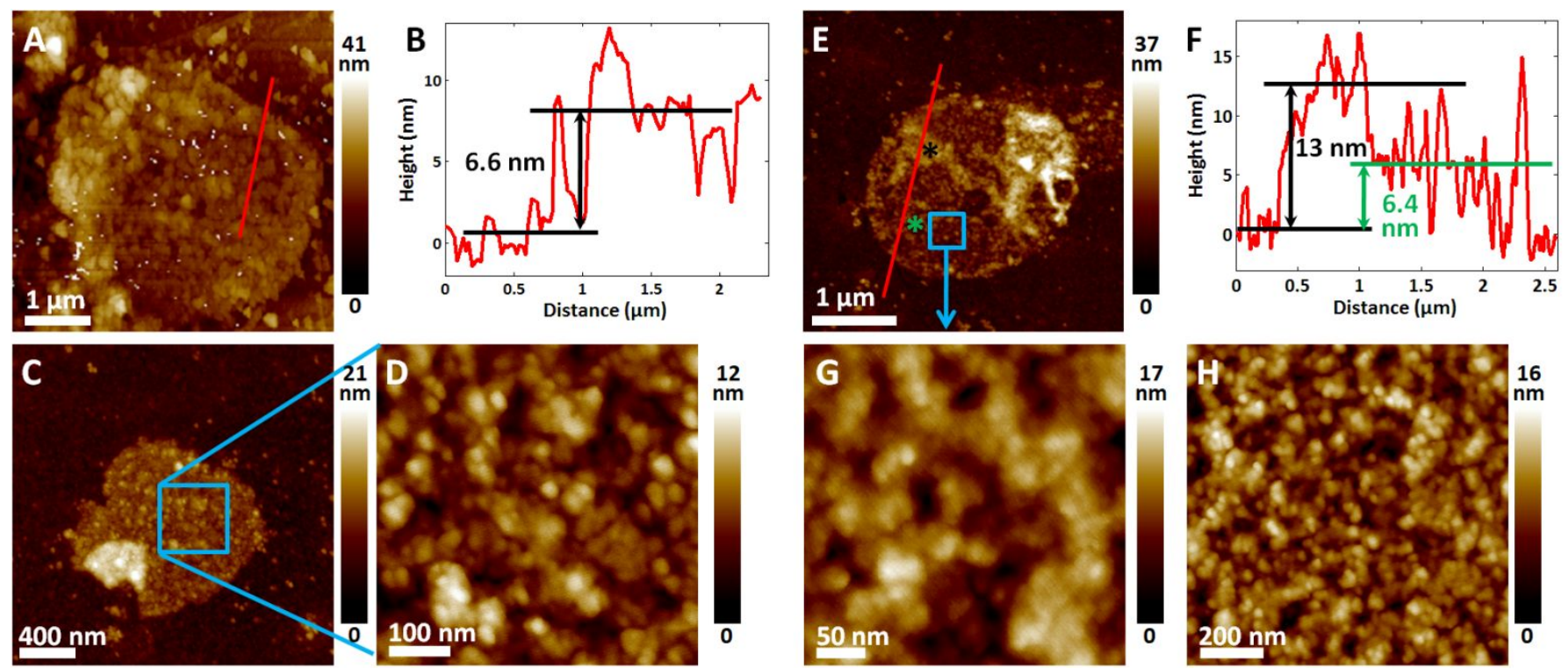

Figure S6 AFM images of membrane patches left on the decellularized glass slides with the use of trypsin. (A, B) HEK293 cell membrane patch. (A) AFM image of HEK293 cell membrane patch and (B) section profile curve taken along the red line in (A). The section profile curve indicate that the thickness of cell membrane patch is about $6.6 \mathrm{~nm}$. (C-D) MCF-7 cell membrane patch. (C) Large scan size imaging, and (D) small scan size imaging. (E, F, G) MCF-7 cell membrane patch. (E) AFM image of a MCF-7 cell membrane patch. The black asterisk denotes that some biological substances attached on the cell membrane, and the green asterisk denotes the cell membrane. (F) Section profile curve taken along the red line in (E), showing that the thickness of cell membrane is about $6.4 \mathrm{~nm}$. (G) Small scan size imaging of cell membrane. 
(H) Another AFM image of the MCF-7 cell membrane patch. The AFM images (D, G, H) show the nanogranular structures on the MCF-7 cell membrane.
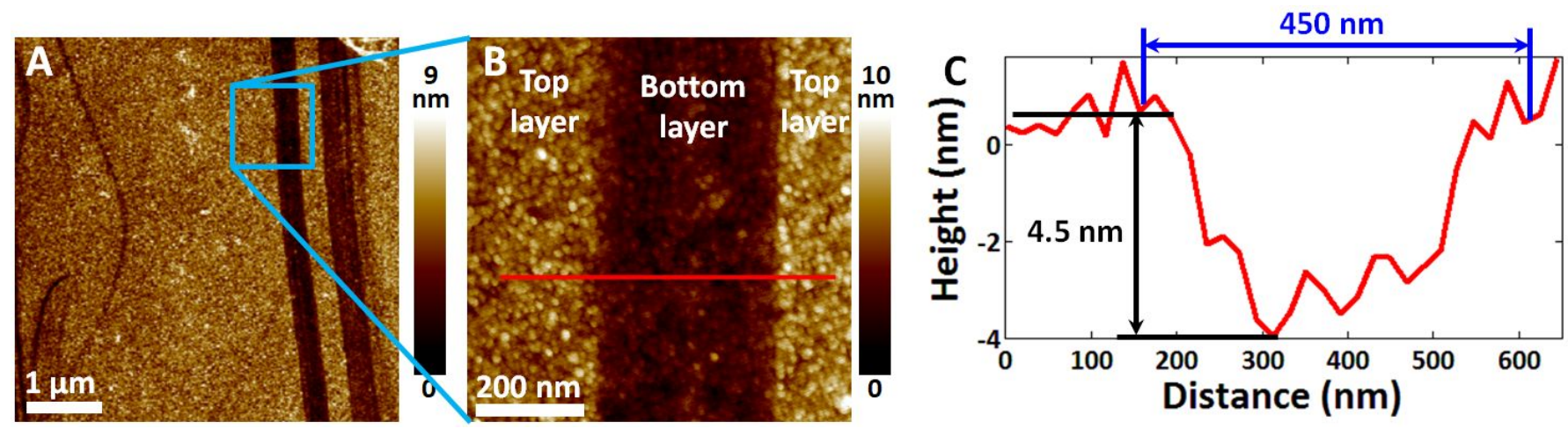

Figure S7 AFM imaging of the multi-layer nanogranular substrates by performing mechanical scratching on the decellularized substrates with the use of micropipette. (A, B) AFM images of the decellularized substrates after mechanical scratching. (A) Large scan size imaging and (B) small scan size imaging, showing the nanogranular structures on both top layer and bottom layer. (C) Section profile curve taken along the red line in (B), showing that the depth of the decellularized substrate caused by micropipette mechanical scratching is about $4.5 \mathrm{~nm}$ and the width of scratching is about $450 \mathrm{~nm}$.

A

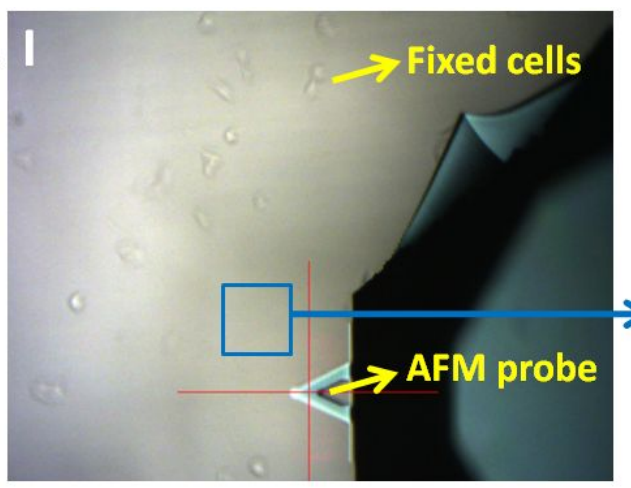

B I

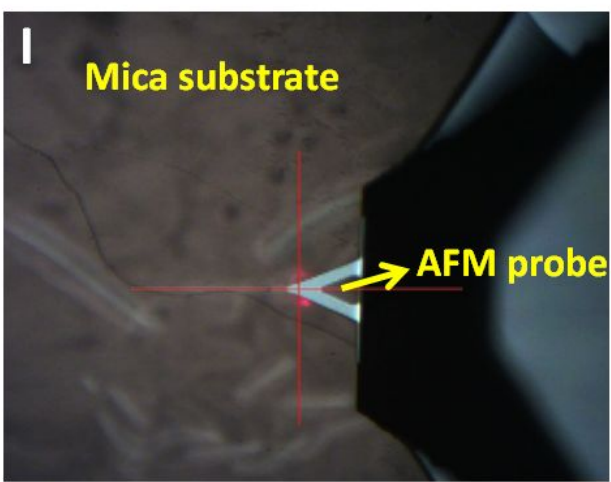

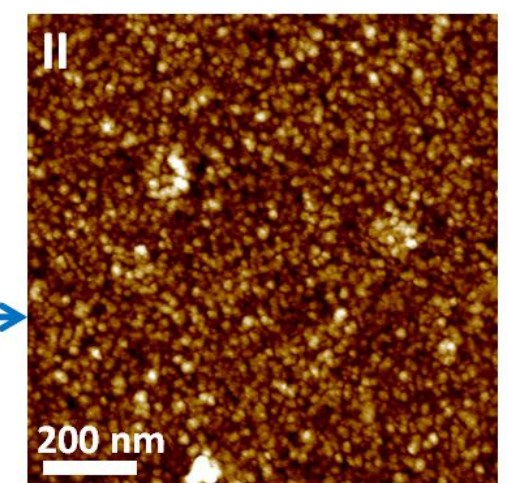
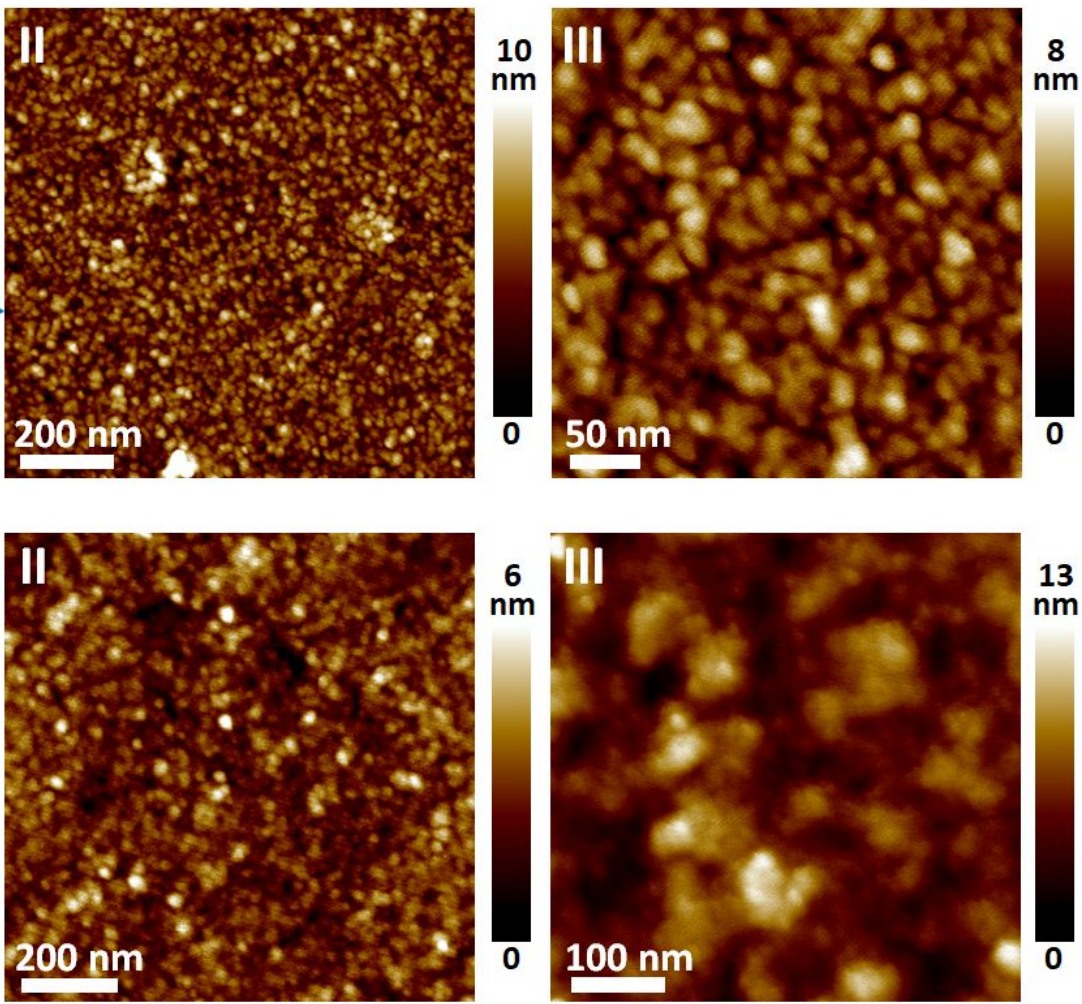

Figure S8 AFM imaging reveals the nanogranular substances from the cell culture medium. (A) AFM imaging of non-cell area of the glass slides on which HEK293 cells were grown. Cells were chemically fixed. (I) Optical image. Non-cell area (denoted by the blue square) was scanned. (II, III) AFM images of the non-cell area of the glass slides. (II) Large-size scan imaging and (III) small-size scan imaging. Nanogranular substances are clearly discernible. (B) AFM imaging of freshly cleaved mica which was placed in cell culture medium at $37^{\circ} \mathrm{C}\left(5 \% \mathrm{CO}_{2}\right)$ for $24 \mathrm{~h}$ without growing cells. (I) Optical image. (II, III) AFM images. (II) Large-size scan imaging and (III) small-size scan imaging. Nanogranular substances are also clearly observed from AFM images. 


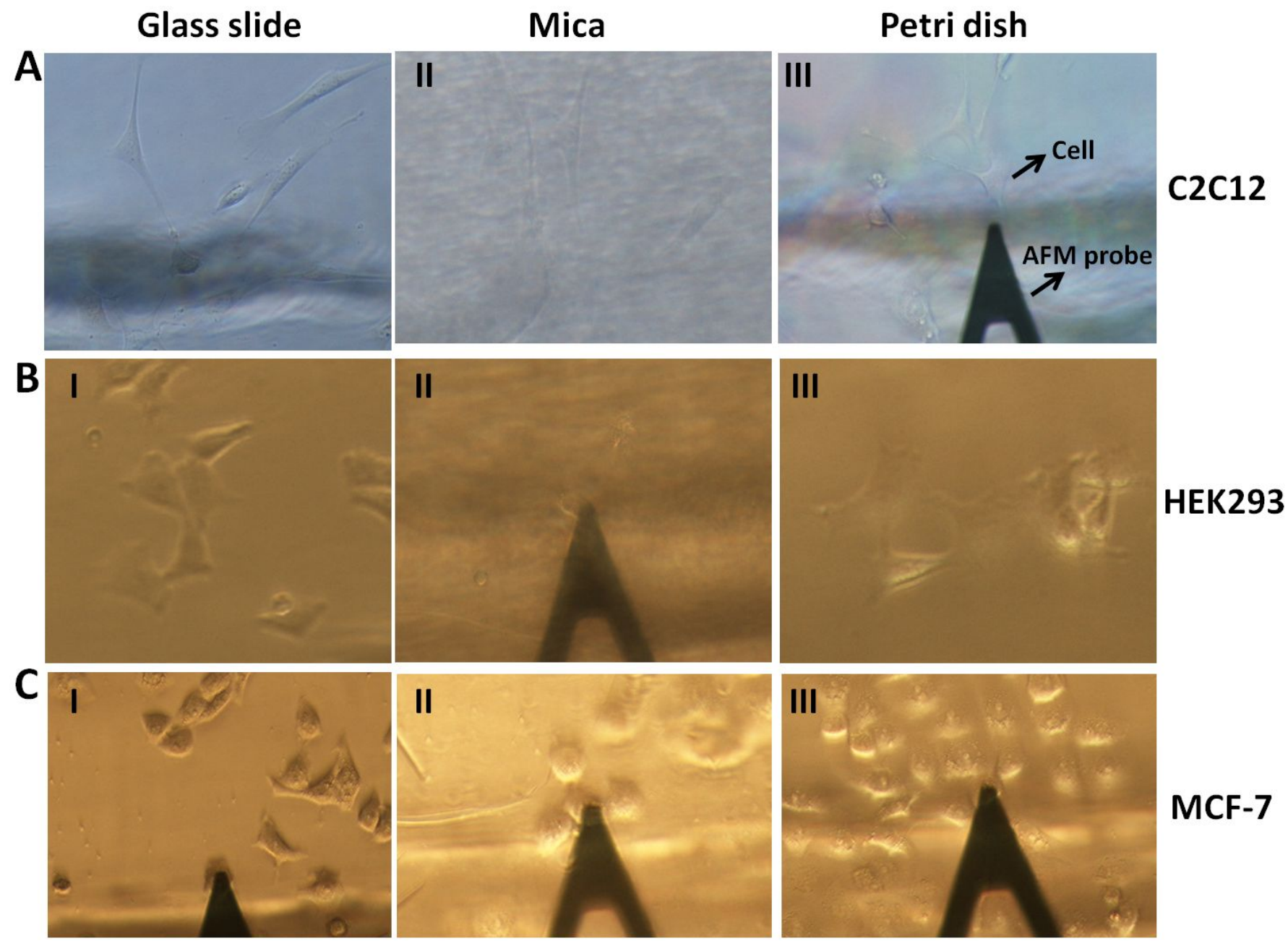

Figure S9 Optical bright field images of moving AFM probe to probe the mechanical properties (adhesion force, Young's modulus, and relaxation time) of living cells grown on different types of substrates in cell culture medium. (A) C2C12 cells. (B) HEK293 cells. (C) MCF-7 cells. (I, II, III) Cells grown on glass slide (I), mica (II), or Petri dish (III). 

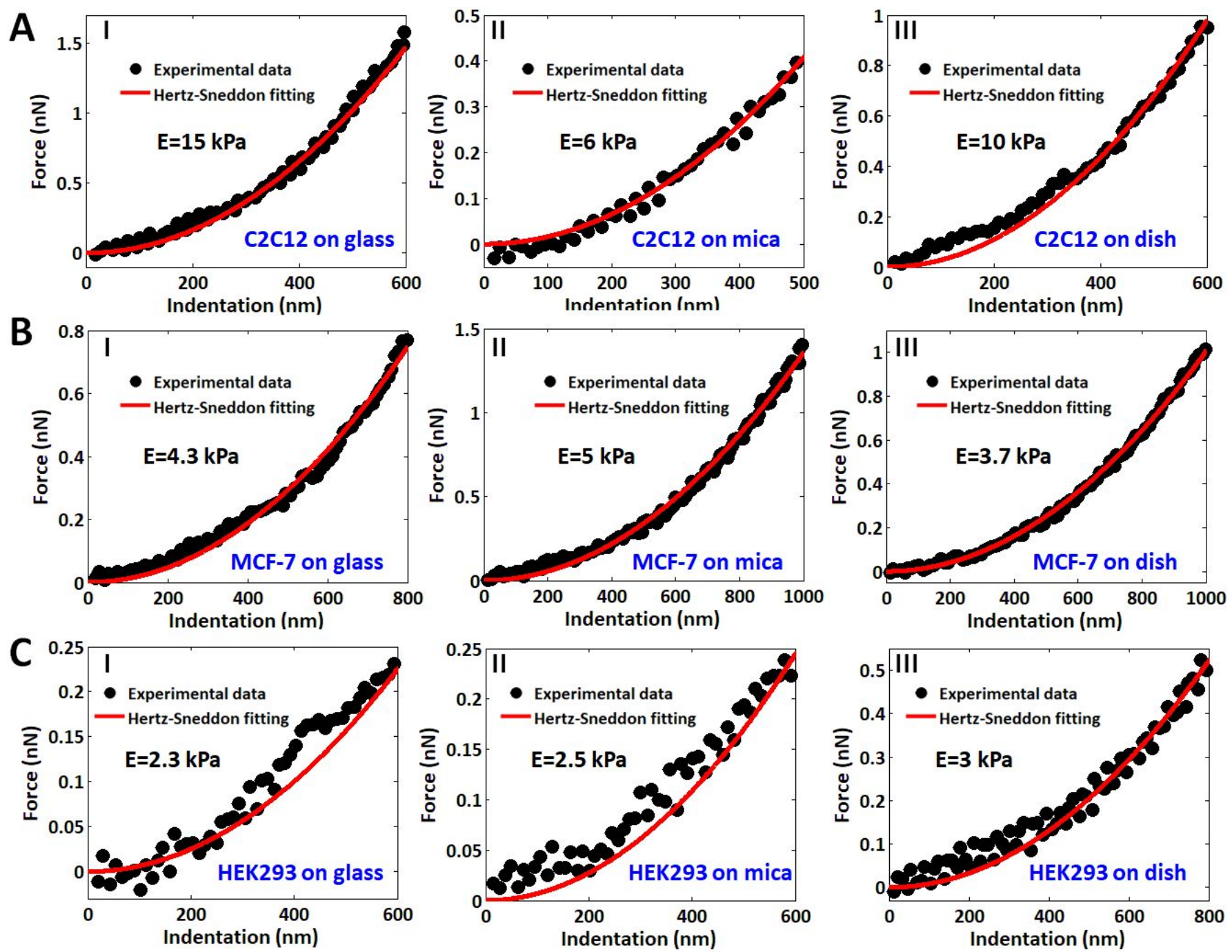

Figure S10 Typical force curves and theoretical fittings of cells on different substrates for characterizing cell elasticity. (A) C2C12 cells. (B) MCF-7 cells. (C) HEK293 cells. (I, II, III) Cells cultured on glass slide (I), mica (II), or Petri dish (II). 

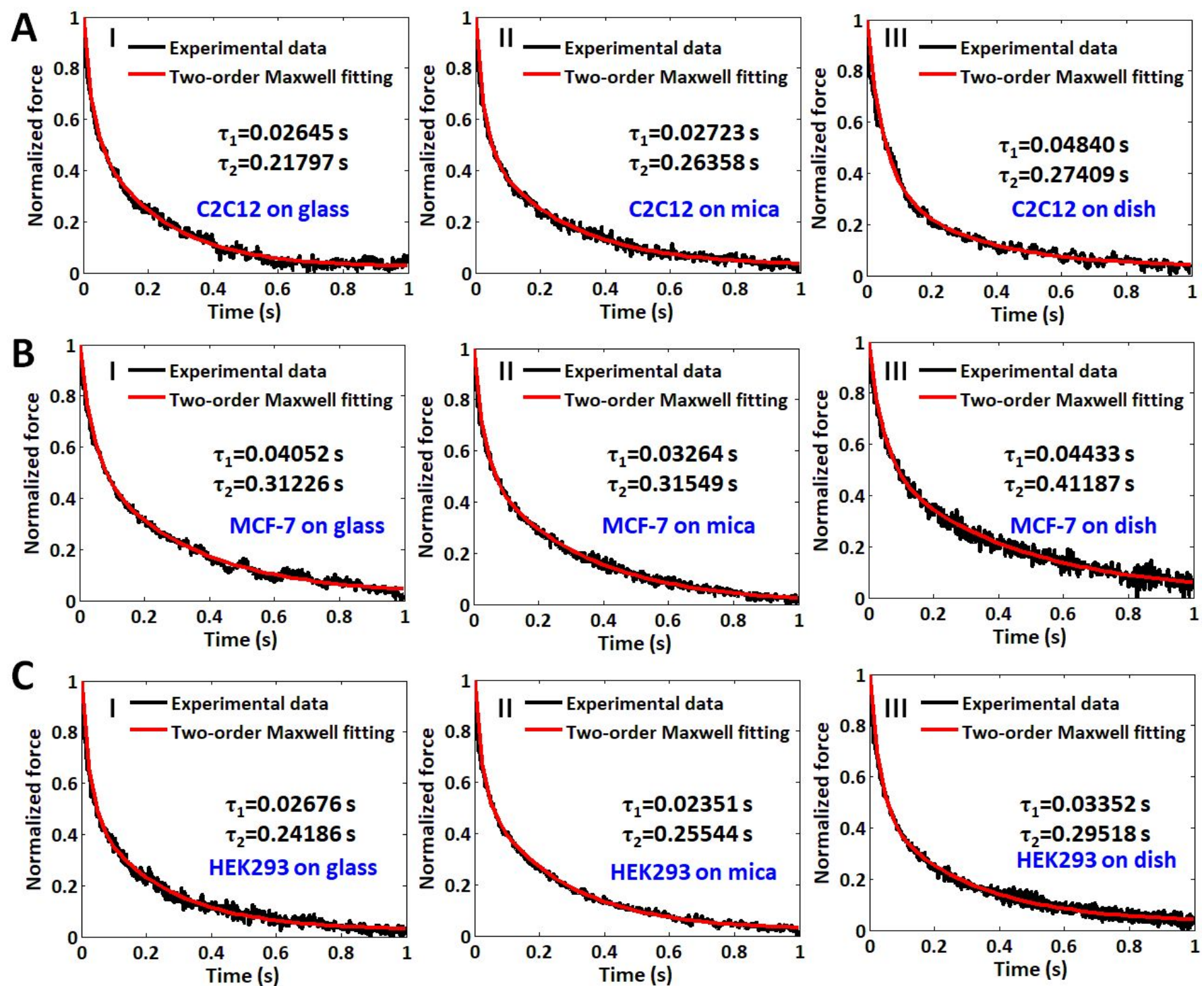

Figure S11 Typical relaxation curves and theoretical fittings of cells on different substrates for characterizing cell viscoelasticity. (A) C2C12 cells. (B) MCF-7 cells. (C) HEK293 cells. (I, II, III) Cells cultured on glass slide (I), mica (II), or Petri dish (II). 